\title{
Representing measures for the disc algebra and for the ball algebra
}

\author{
by RAYMOND BRUMMELHUIS (Madrid) \\ and JAN WiEgERINCK* (Amsterdam)
}

\begin{abstract}
We consider the set of representing measures at 0 for the disc and the ball algebra. The structure of the extreme elements of these sets is investigated. We give particular attention to representing measures for the 2-ball algebra which arise by lifting representing measures for the disc algebra.
\end{abstract}

Introduction. Let $X$ be a compact Hausdorff space, $C(X)$ the algebra of continuous functions on $X$ with the supremum norm, $A$ a function algebra on $X$ and $\Phi$ a multiplicative linear functional on $A$. The set of all probability measures on $X$ which represent $\Phi$ is denoted by $M_{\Phi}$. As is well known, $M_{\Phi}$ is a nonempty, weak*-compact, convex subset of the set of all regular Borel measures, $M(X)$, which is viewed as the dual space of $C(X)$. For a compact $X \subset \mathbb{C}^{n}$, the function algebra $P(X)$ is defined as the closure of the holomorphic polynomials in $C(X)$.

Let $B=B^{n}$ be the unit ball in $\mathbb{C}^{n}, n \geq 2, S$ its boundary. Next, $D$ is the open unit disc in $\mathbb{C}, \bar{D}$ its closure and $T$ its boundary. Traditionally $P(\bar{D})$ is called the disc algebra and denoted by $A(D)$ while $P(S)$ is called the ball algebra and denoted by $A(B)$. Note that by our definition a representing measure for $A(D)$ will be defined on $\bar{D}$, while a representing measure for $A(B)$ will be defined on $S$. The motivation is that in this way we obtain an interesting set of representing measures for $A(D)$, all of which can be "lifted", to furnish representing measures on the sphere. From now on $\Phi$ will be point evaluation at a point $a$, usually 0 , of the unit ball or disc and we will write $M_{a} S$, respectively $M_{a} D$, or when no confusion is possible $M_{a}$, instead of $M_{\Phi}$.

1991 Mathematics Subject Classification: 30H05, 32E25, 46J10, 46J15.

Key words and phrases: ball algebra, disc algebra, extreme point, representing measure.

*Supported by a fellowship of the Royal Netherlands' Academy of Arts and Sciences. 
In this paper we are mainly interested in the extreme points of $M_{0}$ in the case of the ball algebra or the disc algebra. In Section 2 we study $M_{0} D$. A complete description of its extreme points would be very interesting. We have some positive results in this direction. We prove among other things that extreme elements of $M_{0} D$ which have a real-analytic, nontrivial trace on the unit circle, have finite support in $D$. It is observed that this situation can really occur. This answers a question in [Ry] negatively. Various examples of extremal representing measures are given.

Sections 3 and 4 are devoted to $M_{0} S$. This is the case in which we are interested mostly. One reason for this is the Fatou problem for the unit ball, which reads as follows: Let $f$ be a bounded holomorphic function on $B$ and $\mu \in M_{0} S$; does $f$ has radial limits a.e. $[\mu]$ ? We are far from solving this, but we wish to point out that by Choquet's theorem it suffices to solve this problem for extremal elements of $M_{0} S$. Section 3 deals with representing measures for $A\left(B^{2}\right)$ which arise from lifting elements of $M_{0} D$. We give a necessary and sufficient condition under which a lifted measure is extreme. In Section 4 we investigate representing measures on $S$ which arise from embedded discs and prove a density result.

We wish to express our gratitude to John Ryff, who provided us with some useful references, and in particular to Peter de Paepe, who greatly influenced this paper. By our standards he should have been coauthor, but we failed to convince him.

\section{Extreme elements of $M_{0} D$}

2.1. We shall denote the unit point mass ("Dirac measure") at a point $x \in \bar{D}$ by $\delta_{x}$. The representing measures $M_{0} D$ were studied by J. Ryff. He obtained the following result.

TheOREM [Ry]. Let $\mu \in M_{0} D, \mu \neq \delta_{0}$. Then there is a simply connected domain $\Omega \subset D$ that contains the origin, such that $\partial \bar{\Omega} \subset \operatorname{Supp} \mu \subset \bar{\Omega}$ where $\partial \bar{\Omega}$ is the boundary of $\bar{\Omega}$. In fact, $\Omega$ is the component of the origin of the interior of the polynomially convex hull of $\operatorname{Supp} \mu$.

He also observed that with $\Omega \subset D$ as above, harmonic measure on $\partial \Omega$ evaluated at 0 is an extreme element of $M_{0} D$ and asked whether these are the only extreme elements of $M_{0} D$. We shall see shortly that there are much more.

In view of the above result and the Riemann mapping theorem we shall restrict ourselves to the case where $\Omega=D$.

2.2. Definition. Let $\mu$ be a finite real measure on $D$. The Poisson 
transform of $\mu$ is defined by

$$
P_{\mu}(\zeta)=\frac{1}{2 \pi} \iint \frac{|\zeta|^{2}-|z|^{2}}{|z-\zeta|^{2}} d \mu(z)=\frac{1}{2 \pi} \iint \operatorname{Re} \frac{\zeta+z}{\zeta-z} d \mu(z) .
$$

It is easy to see that $P_{\mu}$ is a harmonic function on the complement of the support of $\mu$. Using Fubini's Theorem one shows easily that $\left.P_{\mu}\right|_{T} \in L^{1}(T)$ (compare $[\mathrm{K}]$, p. 336]. If $\mu$ is positive, then so is $P_{\mu}$ on the complement of $D$. If the support of $\mu$ is a compact subset $K$ of $D$, then $P_{\mu}$ is a real analytic function on $T$.

2.3. LEMMA. Let $f \not \equiv 0$ be a nonnegative real-analytic function on the unit circle $T$ and let $\mu$ be a positive finite measure on $D$ such that $\operatorname{Supp} \mu \cap D$ is infinite. Then there exists a simple function $g \not \equiv 0$ supported on a compact subset $K$ of $D$ such that $\left|P_{g \mu}\right| \leq f$.

Proof. Real-analytic functions on $T$ have only finitely many zeros and these are of finite multiplicity. Let $a_{1}, \ldots, a_{k}$ be the zeros of $f$, with multiplicities $l_{1}, \ldots, l_{k}$ respectively. Let $n=1+\sum_{j=1}^{k} l_{j}$. We can find disjoint compact subsets $K_{1}, \ldots, K_{n}$ of $\operatorname{Supp} \mu \cap D$ such that $\mu\left(K_{j}\right)>0, j=1, \ldots, n$. Let $\mu_{j}=\chi_{j} \mu$, where $\chi_{j}$ is the characteristic function of $K_{j}$ and let $F_{j}=P_{\mu_{j}}$. By linear algebra there exist nontrivial $\lambda_{1}, \ldots, \lambda_{n}$ which solve the following system of $n-1$ equations:

$$
\sum_{l=1}^{n} \lambda_{l} F_{l}^{(q)}\left(a_{p}\right)=0, \quad 1 \leq p \leq k, 0 \leq q \leq l_{p}-1 .
$$

Let

$$
g_{\varepsilon}(z)=\varepsilon \sum_{l=1}^{n} \lambda_{l} \chi_{l}(z) .
$$

Note that $P_{g_{\varepsilon} \mu}=\varepsilon \sum_{j=1}^{n} \lambda_{l} F_{l}$. By compactness of $T$ and because $P_{g_{\varepsilon} \mu}$ is a real-analytic function on $T$ which by (1) has at least the same zeros as $f$ (counting multiplicities), there exists a small $\varepsilon$ such that $\left|P_{g_{\varepsilon} \mu}\right| \leq f$, which proves the lemma.

We shall now study $M_{0}=M_{0} D$. First recall that the rotation invariant probability measure $|d \zeta| / 2 \pi$ is the unique representing measure for 0 which is supported on $T$.

2.4. LemmA. Let $\mu \in M_{0}$. Write $\mu=\mu_{1}+\mu_{2}$, where $\mu_{1}$ is concentrated on $D$ and $\mu_{2}$ is concentrated on $T$. Then

$$
\mu_{2}=\left(1 / 2 \pi-P_{\mu_{1}}(\zeta)\right)|d \zeta| \text {. }
$$

In particular, we have $\mu_{2}=s(\zeta)|d \zeta|$ with $s \in L^{\infty}(T), 0 \leq s \leq 1 / 2 \pi$. On the other hand, if $\mu_{1}$ is any positive finite measure and if $\mu_{2}$ defined by (3) is nonnegative, then $\mu=\mu_{1}+\mu_{2} \in M_{0}$. 
Pr o of. Using Fubini's theorem we find, for harmonic polynomials $h$,

$$
\begin{aligned}
h(0) & =\int h d \mu=\int_{T} h d \mu_{2}+\int_{D} h d \mu_{1} \\
& =\int_{T} h d \mu_{2}+\int_{D} \frac{1}{2 \pi} \int_{T} \frac{1-|z|^{2}}{|z-\zeta|^{2}} h(\zeta)|d \zeta| d \mu_{1} \\
& =\int_{T} h d \mu_{2}+\int_{T} h(\zeta) P_{\mu_{1}}(\zeta)|d \zeta| .
\end{aligned}
$$

The right hand side apparently defines a representing measure for 0 concentrated on $T$, which equals $|d \zeta| / 2 \pi$, because of uniqueness. This yields (3). From (3) and the positivity of $\mu_{2}$ and $P_{\mu_{1}}$ we conclude that $s$, which is in $L^{1}(T)$ to begin with, has its values in $[0,1 / 2 \pi]$.

For the last part of the lemma, we compute $\int h d \mu$ as in (4). Combining with (3) we see that $\mu \in M_{0}$.

We shall call the function $s(\zeta)$ the trace of $\mu$ on $T$.

2.5. We shall make use of the following criterion for extremality, which is a special case of Theorem 1 in [D], although the idea seems to go back to Naimark. For convenience of the reader we include the short proof.

TheOrem (Douglas' Criterion). Let $A$ be a function algebra on a compact Hausdorff space $X$, and $\Phi$ a multiplicative continuous linear functional on A. Then

$$
\mu \in M_{\Phi} \text { is an extreme point } \Leftrightarrow \operatorname{Re} A \text { is dense in } L_{\operatorname{Re}}^{1}(\mu) .
$$

Pro of. Suppose that $\mu \in M_{\Phi}$ is not extreme and has nontrivial decomposition $\mu=\left(\mu_{1}+\mu_{2}\right) / 2, \mu_{i} \in M_{\Phi}$. Then the $\mu_{i}$ are positive and absolutely continuous with respect to $\mu$. Hence there exist nonnegative $h_{i} \in L^{1}(\mu)$ with $\mu_{i}=h_{i} \mu$, so that $h_{1}+h_{2}=2, h_{i} \not \equiv 1$. In particular, $h_{i} \in L^{\infty}(\mu)$. Now $1-h_{1}$ is a nontrivial element of $L^{\infty}(\mu) \cong L^{1}(\mu)^{*}$ which annihilates $\operatorname{Re} A$. Therefore $\operatorname{Re} A$ is not dense in $L_{\operatorname{Re}}^{1}(\mu)$. On the other hand, if $\operatorname{Re} A$ is not dense in $L_{\operatorname{Re}}^{1}(\mu)$, then there exists a nontrivial $h \in L^{\infty}(\mu)$ which annihilates $\operatorname{Re} A$. We can assume that $h$ is real-valued and $|h|<1$. Now we can write $\mu=((1+h) \mu+(1-h) \mu) / 2$, which shows that $\mu$ is not extreme.

R e m a r k. Sometimes it will be convenient to complexify the right hand side of (5): Abusing the notation, $\operatorname{Re} A$ will then stand for $\operatorname{Re} A \oplus i \operatorname{Re} A$, and (5) is equivalent to "Re $A$ is dense in $L^{1}(\mu)$ ".

2.6. THEOREM. Let $\mu$ be an extreme element in $M_{0}$ and suppose that the trace of $\mu$ on $T$ is real-analytic and not identically equal to 0 . Then there is a finite subset $K$ of $D$ such that $\operatorname{Supp} \mu=T \cup K$. 
Proof. Let $K=\operatorname{Supp} \mu \cap D$. Suppose $K$ is infinite. Let $\mu_{1}=\left.\mu\right|_{K}$ and let $f$ be the trace of $\mu$ on $T$. By Lemma $2.4, f=1 / 2 \pi-P_{\mu_{1}}$. By Lemma 2.3 there exists a simple function $g \not \equiv 0$ on $K$ such that $\left|P_{g \mu_{1}}\right| \leq f$.

Choose a simple function $\kappa(z)$ on $K$ such that $\int_{K} \kappa(z) g(z) d \mu=1$. We extend $\kappa$ by 0 outside $K$; then $\kappa \in L^{1}(\mu)$. By Douglas' criterion we can approximate $\kappa$ with harmonic polynomials $h$ in $L^{1}(\mu)$. Let $\varepsilon>0$ and let $\int|h-\kappa| d \mu<\varepsilon$; then also $\int_{K}|h-\kappa| d \mu<\varepsilon$ and $\int_{T}|h(\zeta)| f(\zeta) d|\zeta|<\varepsilon$. This implies

$$
\begin{aligned}
1 & =\left|\int_{K} \kappa g d \mu\right| \leq\left|\int_{K} h g d \mu\right|+\|g\|_{\infty} \int_{K}|h-\kappa| d \mu \\
& \leq\left|\int_{T} h P_{g \mu}\right|+\varepsilon\|g\|_{\infty} \leq \int_{T}|h(z) f(z)||d z|+\varepsilon\|g\|_{\infty} \leq \varepsilon+\varepsilon\|g\|_{\infty},
\end{aligned}
$$

which is a contradiction.

2.7. Corollary. Let $\mu$ be an extreme element in $M_{0}$ and suppose that Supp $\mu=T \cup K$, where $K$ is compact in $D$. Then $K$ is finite.

Proof. Let $\chi$ be the characteristic function of $K$. Since $P_{\chi \mu}$ is realanalytic on $T$, so is the trace of $\mu$ on $T$ which equals $1 / 2 \pi-P_{\chi \mu}$, and Theorem 2.6 applies.

Re mark. It follows from the proof that an upper bound for the number of points in the intersection of $D$ and the support of $\mu$ can be read off from the number of zeros of the trace of $\mu$ on $T$. This leads to:

2.8. Example. Suppose $\mu$ is an extreme element of $M_{0}$. If $\operatorname{Supp} \mu \subset$ $\{T \cap[\varepsilon, 1-\varepsilon]\}$, then there exists an $x \in[\varepsilon, 1-\varepsilon]$ such that $\operatorname{Supp} \mu \subset T \cup\{x\}$.

Proof. Easy estimates of the Poisson kernel show that the trace of $\mu$ on $T$ has at most a zero of order 2 at 1 . Also for every subset $K$ of $[\varepsilon, 1-\varepsilon]$ with $\mu(K) \neq 0$ and putting $\chi=\chi_{K}, P_{\chi \mu}$ will have a maximum at 1 , and an inequality like the one in Lemma 2.3 can be obtained using only two subsets of $[\varepsilon, 1-\varepsilon]$. Now one copies the proof of Theorem 2.6.

Next we shall give some examples of more or less pathological representing measures. The first one answers Ryff's question negatively:

2.9. Example. Let $x \in D, x \neq 0$. There exists an extreme element of $M_{0}$ with support equal to $T \cup\{x\}$.

Proof. Obviously, if $\mu \in M_{0}$ is supported on $T \cup\{x\}$, then $\mu=\varepsilon \delta_{x}+$ (trace of $\mu$ ) $d|\zeta| / 2 \pi$. Hence by Lemma 2.4, for small enough $\varepsilon>0, \mu_{\varepsilon}=$ $d|\zeta| / 2 \pi-\varepsilon P_{\delta_{x}}(\zeta) d|\zeta|+\varepsilon \delta_{x}$ will be a representing measure with support $T \cup\{x\}$. Applying the Krein-Milman theorem to the set of representing measures supported on $T \cup\{x\}$, we can find an extreme element of $M_{0}$ 
with support containing $x$. In fact, this extreme element is $\mu_{\varepsilon_{0}}$, where $\varepsilon_{0}=\max _{\varepsilon>0}\left\{\varepsilon: 1 / 2 \pi-\varepsilon P_{\delta_{x}} \geq 0\right\}$. One can check Douglas' criterion explicitly for $\mu_{\varepsilon_{0}}$. Note that the measures $\mu_{\varepsilon}$ are representing measures which are not Jensen measures: $\log |x|>\int \log |z-x| d \mu_{\varepsilon}$.

2.10. The following example shows that there are extreme elements of $M_{0}$ of quite different nature. At least part of it, if not all, has been known. We could not find a reference for the observation about extreme elements in this example.

Recall that since $C(X)$ is separable, the unit ball in $C(X)^{*}$ is metrizable and so is $M_{0}$. Hence we may apply Choquet's theorem (cf. $[\mathrm{A}]$ or $[\mathrm{P}]$ ) which reads as follows.

THEOREM (Choquet). Every point $q$ in a metrizable compact convex set $K$ can be represented as $q=\int \operatorname{Id}(x) d \tau(x)$, where $\operatorname{Id}$ is the identity mapping on $K$ and $\tau$ is a probability measure concentrated on the $G_{\delta}$-set consisting of the extreme points of $K$.

Thus, for every affine continuous function $f$ on $K$, we have $f(q)=\int f d \tau$. In fact, it is another theorem of Choquet that the latter statement is also true if $f$ is only an affine function of the first Baire class, that is, a pointwise limit of continuous functions, on $X$ (cf. [P, p. 100]).

EXAMPLE. Let $\mathcal{A}=\left\{a_{n}\right\}$ be a sequence of distinct points in $D$ without interior limit points. Suppose that almost every point of $T$ is a nontangential limit point of $\mathcal{A}$. Theorem 4 of [BSZ] states that for every $z \in D$ there exists a (complex) representing measure $\mu \in M_{z}$ which is concentrated on $\mathcal{A}$. Next by Theorem 2.2 in [G, Ch. 2] (see also [HR]) there exists a positive $f \in L^{1}(|\mu|)$ with $f|\mu| \in M_{0}$. Obviously, $f|\mu|=\sum c_{j} \delta_{a_{j}}$, where $c_{j} \geq 0$. Selecting a subsequence $\mathcal{A}^{\prime}$ we may asume $c_{j}>0$, while still $T \subset \overline{\mathcal{A}^{\prime}}$, since otherwise 0 would not be in the polynomially convex hull of $\overline{\mathcal{A}^{\prime}}$. Now we use Choquet's theorem. This gives for $f|\mu|$ the existence of a probability measure $\tau=\tau_{f|\mu|}$ which is concentrated on the set of extreme points $E$ of $M_{0}$, and for all affine functions $g$ of the first Baire class on $M_{0}$ the representation

$$
g(f|\mu|)=\int_{M_{0}} g d \tau .
$$

Let $\chi$ denote the characteristic function of $\mathcal{A}^{\prime}$. This gives rise to an affine function $\chi^{*}$ on $M_{0}$, defined by $\chi^{*}(\nu)=\int_{D} \chi d \nu$. It is easily seen that $\chi^{*}$ is a pointwise limit of continuous functions on $M_{0}$. Thus we obtain

$$
1=\chi^{*}(f|\mu|)=\int_{E} \chi^{*} d \tau .
$$


Since $\chi^{*} \leq 1$ on $M_{0}$, it follows that $\chi^{*}(\nu)=1$ a.e. $\tau$, in other words, since $\tau$ is concentrated on $E, \int_{D} \chi d \nu=1$ a.e. $\tau$. We infer that there exist extreme elements of $M_{0}$ concentrated on $\mathcal{A}^{\prime}$.

EXAMPLE. There exist representing measures in $M_{0}$ which are concentrated on a countable dense subset of $D$.

Proof. Pick a countable dense sequence of circles $\left\{T_{j}\right\}$, all centered at 0 , and a sequence $\left\{b_{j}\right\}$ such that $b_{j}>0$ and $\sum b_{j}=1$. By scaling the previous example, there exists $\mu_{j} \in M_{0}$ concentrated on a countable set with limit points $T_{j}$. Now $\sum b_{j} \mu_{j}$ is the required measure.

The above results lead us to

2.11. Conjecture. Let $\mu \in M_{0}$ be extreme and $T \subset \operatorname{Supp} \mu$. Then Supp $\mu \cap D$ is a discrete subset of $D$.

Some additional support for this conjecture is given by the following.

2.12. Proposition. If $\mu$ is extreme, with Lebesgue decomposition $\mu=$ $f \lambda+\nu$ with respect to Lebesgue measure $\lambda$, then $f \geq c>0$ is impossible on an open set.

P r o of. Indeed, if not, then $f$ would be positive on two tiny, concentric annuli $A_{1}, A_{2}$. It is easy to see that $\mu$ is a convex combination of $\mu_{1}, \mu_{2}$, where $\mu_{i}$ is obtained by replacing $f$ by $f \pm \varepsilon \chi_{1} \mp \delta \chi_{2}$, and $\varepsilon$ and $\delta$ are suitably chosen positive numbers, while $\chi_{i}$ is the characteristic function of $A_{i}$.

2.13. Recall that $\mu \in M_{0}$ has minimal support if $\nu \in M_{0}$, Supp $\nu \subseteq$ $\operatorname{Supp} \mu \Rightarrow \operatorname{Supp} \nu=\operatorname{Supp} \mu$. At first glance one might think that minimal support and extremality are connected. Indeed, for the disc algebra it follows from Ryff's theorem that a representing measure with minimal support is harmonic measure for some domain and therefore extreme. On the other hand, Example 2.9 also provides an example of an extreme element of $M_{0}$ which does not have minimal support. For other algebras an example of a representing measure with minimal support but not extreme is quite easy.

ExAmple. Let $A^{0}(D)$ be the function algebra on $T$ spanned by $\left\{z^{2}, z^{3}\right\}$. For $f \in A^{0}(D)$, we have

$$
\int_{T} f(z) \frac{d z}{2 \pi i z}=f(0), \quad \int_{T} f(z) d z=0, \quad \int_{T} f(z) \frac{d z}{2 \pi i z^{2}}=0 .
$$

On parametrizing $T$ with $e^{i \theta}$, the last two equations give

$$
\int_{-\pi}^{\pi} f\left(e^{i \theta}\right) \cos \theta d \theta=0
$$


The conclusion is that for $-1 \leq \alpha \leq 1,(1+\alpha \cos \theta) d \theta / 2 \pi$ is a representing measure with minimal support. It is, however, not an extreme element for $-1<\alpha<1$.

This example will be modified in the sequel to provide a similar example for $A(B)$.

\section{Circular representing measures}

3.1. There is a natural way to pass from representing measures for $A(D)$ to representing measures for $A(B)$ and vice versa (cf. [R]). In fact, let $m \in M_{0} D$. We consider $D$ as $B \cap\left\{z_{2}=0, \ldots, z_{n}=0\right\}$ and define $m^{C}$ on $S$ as follows. For any continuous $v$ on $S$,

$$
\int_{S} v d m^{C}:=\int_{\bar{D}} d m\left(z_{1}\right) \int_{S^{\prime}} v\left(z_{1}, \sqrt{1-\left|z_{1}\right|^{2}} \zeta^{\prime}\right) d \sigma\left(\zeta^{\prime}\right),
$$

where $\sigma\left(z^{\prime}\right)$ denotes the rotation invariant probability measure on the sphere $S^{\prime}$ in the $z^{\prime}=\left(z_{2}, \ldots, z_{n}\right)$ space. This process will be called lifting $m$ and $m^{C}$ will be called a circular measure.

On the other hand, if $m^{C} \in M_{0} S$, then we define $m \in M_{0} D$ by

$$
\int_{\bar{D}} v d m:=\int_{S} v d m^{C}
$$

for all $v \in C(\bar{D})$ (on the right hand side $v$ is considered as a function on $S$, only depending on the first variable). This will be called projecting $m^{C}$.

Of course we have a similar lifting procedure relating representing measures supported on a (full) ball in $\mathbb{C}^{k}$ to representing measures supported on the sphere in $\mathbb{C}^{k+j}$. In the sequel we shall mainly study the case where $B=B^{2}$ is the unit ball in $\mathbb{C}^{2}$. What happens if one circles an extreme element of $M_{0} D$ ?

3.2. TheOREM. Let $\mu \in M_{0} D$ and $S$ the unit sphere in $\mathbb{C}^{2}$, put $r(z)=$ $\sqrt{1-|z|^{2}}$. The measure $\mu^{C}$ obtained by lifting $\mu$ is extreme in $M_{0} S$ if and only if $\mu$ is extreme in $M_{0} D$ and the holomorphic polynomials are dense in $L^{1}(D, r(z) \mu(z))$.

Proof. Let $z, w$ be the coordinates in $\mathbb{C}^{2}$ and view $D$ as $B \cap\{w=0\}$.

Suppose $\mu^{C}$ is extreme. If $\mu=\frac{1}{2} \mu_{1}+\frac{1}{2} \mu_{2}, \mu_{i} \in M_{0} D$, then $\mu^{C}=$ $\frac{1}{2} \mu_{1}^{C}+\frac{1}{2} \mu_{2}^{C}$, hence $\mu_{i}^{C}=\mu^{C}$, therefore, $\mu_{i}=\mu$ and $\mu$ is extreme. Next we apply Douglas' criterion. If $f \in L^{1}(D, r(z) \mu(z))$, then $f w \in L^{1}\left(S, \mu^{C}\right)$, so for every $\varepsilon>0$ there exists a pluriharmonic polynomial $P(z, w)$ (which may be complex-valued!) with $\int|f(z) w-P(z, w)| d \mu^{C}<\varepsilon$. Expand

$$
P(z, w)=P_{0}(z)+\sum_{j=1}^{N} P_{j}(z) w^{j}+\sum_{j=-N}^{-1} \overline{P_{j}(z)} \bar{w}^{j},
$$


where $P_{0}$ is a harmonic polynomial and $P_{j}$ are holomorphic polynomials if $j \neq 0$. Now, with $w=|w| e^{i \phi}=r(z) e^{i \phi}$ on $S$, we have

$$
\begin{aligned}
\int\left|f-P_{1}\right| r(z) d \mu(z) & =\iint\left|f(z)-P_{1}(z)\right| \frac{d \phi}{2 \pi} r(z) d \mu(z) \\
& =\int\left|\int \frac{f(z) w-P(z, w)}{w} \frac{d \phi}{2 \pi}\right| r(z) d \mu(z) \\
& \leq \iint|f(z) w-P(z, w)| \frac{d \phi}{2 \pi} d \mu(z) \\
& =\int|f(z) w-P(z, w)| d \mu^{C}<\varepsilon .
\end{aligned}
$$

For the converse implication, again by Douglas' criterion, it suffices to show that continuous functions can be approximated by pluriharmonic polynomials in $L^{1}\left(S, \mu^{C}\right)$. Let $f$ be continuous on $S$ and $\varepsilon>0$. Let

$$
f_{1}= \begin{cases}f & \text { on } S \backslash\{|z|=1\} \\ 0 & \text { on }|z|=1\end{cases}
$$

The function $f-f_{1}$ is independent of $w$ and can be viewed as a function on $\bar{D}$, supported on $T$, which is in $L^{1}(\mu)$. There exists a harmonic polynomial $P_{1} \in \operatorname{Re} A(D)$ with

$$
\int_{\bar{D}}\left|f-f_{1}-P_{1}\right| d \mu=\int_{S}\left|f-f_{1}-P_{1}\right| d \mu^{C}<\varepsilon .
$$

Take $\delta>0$ so small that

$$
\int_{1-\delta<|z| \leq 1}\left|f_{1}\right| d \mu^{C}=\int_{1-\delta<|z|<1}|f| d \mu^{C}<\varepsilon
$$

and let $\chi \in C_{0}^{\infty}(D)$ with $0 \leq \chi \leq 1, \chi \equiv 1$ if $|z|<1-\delta$. Put $g=\chi f$. Let $F_{N}$ be the Fejér kernel of order $N$. For large enough $N$ we have on $D$

$$
\left\|g\left(z, r(z) e^{i \phi}\right)-g * F_{N}\left(z, r(z) e^{i \phi}\right)\right\|_{\infty}<\varepsilon .
$$

Here we convolve with respect to $\phi$. With

$$
\widehat{g}_{N}(z, k)=(1-|k| / N) \int g\left(z, r(z) e^{i \phi}\right) e^{-i k \phi} \frac{d \phi}{2 \pi},
$$

and observing that $r(z)^{|k|} e^{i k \phi}$ is just the restriction to $S$ of $w^{k}$ if $k>0$, and of $\bar{w}^{|k|}$ if $k<0$, we have

$$
\begin{aligned}
g * F_{N}\left(z, r(z) e^{i \phi}\right) & =\sum_{|k| \leq N} \widehat{g}_{N}(z, k) e^{i k \phi}=\sum \frac{\widehat{g}_{N}(z, k)}{r(z)^{|k|}} r(z)^{|k|} e^{i k \phi} \\
& =c_{0}(z)+\sum_{k=1}^{N} c_{k}(z) w^{k}+\sum_{k=-N}^{-1} \overline{c_{k}(z)} \bar{w}^{|k|} .
\end{aligned}
$$


Here the $c_{k}$ are defined by the last equality. As $\widehat{g}_{N} \equiv 0$ if $r(z)$ is small enough, it follows that the $c_{k}$ are continuous and that $\operatorname{Supp} c_{k} \Subset D$. Now there exists a harmonic polynomial $Q_{0}(z, \bar{z})$ with $\int\left|c_{0}-Q_{0}\right| d \mu(z)<\varepsilon$ and for $k \neq 0, c_{k}$ can be approximated in $L^{1}(D, r(z) \mu)$ by holomorphic polynomials. Thus there exist holomorphic polynomials $Q_{k}(z)$ with

$$
\int\left|c_{k}-Q_{k}\right| r d \mu<\varepsilon / 2^{|k|} \text {. }
$$

But then

$$
\int\left|c_{k} w^{|k|}-Q_{k} w^{|k|}\right| d \mu^{C}=\int\left|c_{k}-Q_{k}\right| r^{|k|} d \mu \leq \int\left|c_{k}-Q_{k}\right| r d \mu<\varepsilon / 2^{|k|} .
$$

We put

$$
P_{2}(z, w)=Q_{0}(z)+\sum_{k=1}^{N} Q_{k}(z) w^{k}+\sum_{k=-N}^{-1} \overline{Q_{k}(z)} \bar{w}^{|k|}
$$

and conclude that $P_{2} \in \operatorname{Re} P(S)$ such that $\int_{S}\left|\chi f * F_{N}-P_{2}\right| d \mu^{C}<3 \varepsilon$. Now

$$
\begin{aligned}
\int\left|f-P_{1}-P_{2}\right| d \mu^{C} \leq & \int\left|f-f_{1}-P_{1}\right| d \mu^{C}+\int\left|f_{1}-\chi f\right| d \mu^{C} \\
& +\int\left|\chi f-\chi f * F_{N}\right| d \mu^{C}+\int\left|g * F_{N}-P_{2}\right| d \mu^{C} \\
\leq & 6 \varepsilon .
\end{aligned}
$$

3.3. Remarks. A typical situation of the theorem arises as follows. Consider the curve $\Gamma=\Gamma_{1} \cup \Gamma_{2}$ in $\mathbb{C}$ defined by

$$
\begin{aligned}
& \Gamma_{1}=\left\{z \in \mathbb{C}:|z|^{2}=1, \operatorname{Re} z \leq 1 / 2\right\}, \\
& \Gamma_{2}=\{z=x+i y \in \mathbb{C}: x=1 / 2,-\sqrt{3 / 4} \leq y \leq \sqrt{3 / 4}\} .
\end{aligned}
$$

Let $\mu_{0}$ be harmonic measure with respect to 0 on $\Gamma$. As is well known, $\mu_{0}$ is absolutely continuous with respect to arc length. Let $\mu^{C}$ be the measure on $S$ obtained by lifting $\mu_{0}$. Since the polynomials on $\Gamma$ generate a Dirichlet algebra, that is, $\operatorname{Re} P(\Gamma)$ is dense in $C(\Gamma), \mu_{0}$ is the unique representing measure on $\Gamma$ and therefore extreme. From the Weierstrass approximation theorem we conclude that the polynomials are dense in $L^{1}\left(\Gamma_{2}, \mu_{0}\right)$ so it follows from Theorem 3.2 that $\mu^{C}$ is extreme in $M_{0} S$.

Note that the support of $\mu^{C}$ is equal to $K=K_{1} \cup K_{2}$, where

$$
\begin{aligned}
& K_{1}=\Gamma_{1} \times\{0\}, \\
& K_{2}=\left\{\left(1 / 2+i y, R(y) e^{i \phi}\right): 0 \leq \phi \leq 2 \pi,-\sqrt{3 / 4} \leq y \leq \sqrt{3 / 4}\right\}
\end{aligned}
$$

and $R(y)=\sqrt{3 / 4-y^{2}}$. Thus the support of $\mu^{C}$ is a set of positive 2dimensional Hausdorff measure $m_{2}$ and moreover $\mu^{C}$ is not singular with respect to $m_{2}$.

Instead of the interval $\Gamma_{2}$ and the arc $\Gamma_{1}$ we can take any Jordan arc $\gamma_{2} \subset D, 0 \notin \gamma_{2}$, with different end points on $T$, together with a subarc $\gamma_{1}$ of $T$ which connects the endpoints of $\gamma_{2}$, such that 0 lies in the bounded 
component of the complement of the closed Jordan curve $\gamma=\gamma_{1} \cup \gamma_{2}$. We consider harmonic measure $\mu_{0}$ with respect to 0 on $\gamma$. Lifting in the same way as above we find a compact $K \subset S$ and $\mu^{C} \in M_{0}$ with support $K$. Using Walsh' theorem instead of Weierstrass', we find again that $\mu^{C}$ is extreme.

In particular, we can take for $\gamma_{2}$ an arc of positive 2-dimensional measure. Then $\sigma(K)>0$, so we have obtained an extreme representing measure whose support has positive invariant measure. However, in view of the sequel we remark that by a theorem in $[\varnothing] \mu_{0} \perp$ Lebesgue measure in the plane and hence $\mu \perp \sigma$. In fact, it was shown recently in [JW] that, under much more general conditions, $\mu_{0} \perp m_{d}$, where $m_{d}(d>1)$ is $d$-dimensional Hausdorff measure.

Theorem 3.2 also readily gives an explicit $\mu^{C} \in M_{0} S$ which is extreme and not Jensen: Take $\mu$ as in Example 2.9 and lift.

In addition we mention the following result:

3.4. Theorem. Let $\mu \in M_{0} D$ be extreme and $K=$ Supp $\mu^{C}$. If $F=$ Supp $\mu \cap D$ is polynomially convex and has empty interior, then $P(K)$ is a Dirichlet algebra.

Indication of proof. Mergelyan's theorem gives that $P(F)=$ $C(F)$. Now one can repeat the converse part of the proof of Theorem 3.2 with uniform instead of $L^{1}$ estimates.

The situation where $\mu$ is harmonic measure on a smooth curve can be handled very well. The next theorem states roughly that $\mu^{C}$ is extreme if and only if the curve has points of extremely high contact with $T$, say like $e^{-1 /|x|}$ touches the $x$-axis. The fact that (ii) and (iii) in the next theorem are equivalent is a direct consequence of harmonic measure and arc length being comparable. This result is well known in a much more general setting. We include the proof for convenience of the reader.

3.5. TheOREM. Let $\Gamma$ be a $C^{2}$ Jordan curve around 0 in $\bar{D}$ which bounds a domain $\Omega$ and let $\mu$ be harmonic measure with respect to 0 for $\Omega$. Then the following are equivalent:

(i) $\mu^{C}$ is extreme.

(ii) $\log r(z) \notin L^{1}(\mu)$.

(iii) $\log r(z) \notin L^{1}(\Gamma, d s)$, where ds denotes arc length on $\Gamma$.

Proof. Let $F$ denote a conformal map from $D$ to $\Omega$ such that $F(0)=$ 0 . As is well known, $F$ extends to diffeomorphism from $\bar{D}$ to $\bar{\Omega}$, and by invariance of harmonic measure under $F,|d \zeta| / 2 \pi=F^{*} \mu$, the pull-back of $\mu$ under $F$, and also $\mu(z)=\left|\left(f^{-1}\right)^{\prime}(z)\right| d s$, therefore (ii) and (iii) are equivalent. 
Now to show that (i) and (ii) are equivalent, first note that $\mu$ is extreme in $M_{0} D$. By Theorem 3.2, (i) is then equivalent to the holomorphic polynomials being dense in $L^{1}(r(z) \mu)$. Suppose that this were not the case. Then:

The holomorphic polynomials $P$ are not dense in $L^{1}(r(z) \mu) \Leftrightarrow$ $\exists h \in L^{\infty}(r(z) \mu), h \not \equiv 0$, such that

$$
0=\int_{\Gamma} P(z) h(z) r(z) d \mu(z)=\int_{T} P \circ F(\zeta) h \circ F(\zeta) r \circ F(\zeta) \frac{|d \zeta|}{2 \pi}, \quad \forall P .
$$

Observe that $\zeta^{n}$ is a uniform limit on $T$ of functions of the form $P \circ F(\zeta)$ and vice versa. Hence the last statement is equivalent to

$$
\exists h^{\prime} \in L^{\infty}(T) \quad \text { such that } \quad \int \zeta^{n} h^{\prime}(\zeta) r \circ F(\zeta) \frac{|d \zeta|}{2 \pi}=0, \quad n \geq 0 .
$$

As is well known (see e.g. [Gar]), this is equivalent to

$$
\exists h^{\prime} \in L^{\infty}(T) \text { such that } h^{\prime} r \circ F \in H^{\infty},
$$

and by a theorem of F. Riesz (cf. [G]) this is the case if and only if $\exists h^{\prime} \in$ $L^{\infty}(T)$ such that $\log \left|h^{\prime} r \circ F\right|$ is integrable. Since $h^{\prime}$ must be bounded and $r<1$, this reduces to $\int \log r \circ F|d \zeta| / 2 \pi>-\infty$ or $\log r(z) \in L^{1}(\mu)$. Hence we have shown that (i) and (ii) are equivalent.

In general, the condition that the polynomials be dense in $L^{1}(D, r(z) \mu)$ seems only poorly understood. This motivates the following result.

3.6. TheOrem. Let $\mu \in M_{0} D$. Suppose that there exists $F \in A(D)$ such that

$$
|F(z)|=O\left(\sqrt{1-|z|^{2}}\right), \quad z \in \operatorname{Supp} \mu,|z| \rightarrow 1 .
$$

Then $\mu^{C}$ is not extremal.

Proof. Let

$$
h(z, w)=\operatorname{Re}\left(\frac{z F(z) \bar{w}}{1-|z|^{2}}\right)
$$

$F$ as in the theorem. We claim that $h \perp \operatorname{Re} A(D)$ in $L^{2}\left(\mu^{C}\right)$. Since $h$ is real, it suffices to show that $h \perp A(D)$. For this one just notes that, for all $k, l \in \mathbb{N}$,

$$
\int\left(\frac{\overline{z F(z)} w}{1-|z|^{2}}\right) z^{k} w^{l} d \mu^{C}=0
$$


and, again with $r(z)=\left(1-|z|^{2}\right)^{1 / 2}$,

$$
\begin{gathered}
\int\left(\frac{z F(z) \bar{w}}{1-|z|^{2}}\right) z^{k} w^{l} d \mu^{C}=\int d \mu(z) \frac{z^{k+1} F(z)}{r(z)^{2}} r(z)^{l+1} \int_{0}^{2 \pi} e^{i(l-1) \theta} \frac{d \theta}{2 \pi} \\
= \begin{cases}0 & \text { if } l \neq 1, \\
\int z^{k+1} F(z) d \mu(z)=0 & \text { if } l=1, \text { since } \mu \text { is representing. }\end{cases}
\end{gathered}
$$

Finally, $\left|h\left(z, r(z) e^{i \phi}\right)\right| \leq|F(z)| / \sqrt{1-|z|^{2}}$, which is bounded on Supp $\mu$ by the assumptions. So $h \in L_{\mathrm{Re}}^{\infty}\left(\mu^{C}\right)$ and $h$ annihilates $\operatorname{Re} A(D)$ with respect to $\mu^{C}$. Hence $\operatorname{Re} A(D)$ is not dense in $L^{1}\left(\mu^{C}\right)$. By Douglas' criterion we are done.

We now return to $\mathbb{C}^{n}$. Again $\sigma$ is normalized rotation invariant measure on $S$. In view of Proposition 2.2 we see that if $\mu^{C}=f \sigma+\nu, \nu \perp \sigma$, is extreme, then $f$ cannot be bigger than a positive constant on an open set. This is true for all extreme elements of $M_{0} S$.

3.7. TheOREM. Let $\mu \in M_{0} A(B)$. Suppose that $\mu=f \sigma+\nu$, where $\nu \perp \sigma$ and $f \in L^{1}(\sigma), f \geq c>0$ on an open subset of $S$. Then $\mu$ is not extreme in $M_{0}$.

Proof. We will apply Douglas' criterion. Let $E \subset S$ be open and such that $f \geq c>0$ on $E$. Then we have, for every $g \in C(S), P \in \operatorname{Re} A(B)$,

$$
\int_{S}|g-P| d \mu \geq \frac{1}{c} \int_{E}|g-P| d \sigma .
$$

Hence it suffices to prove that $\operatorname{Re} A(B)$ is not dense in $L^{1}\left(\chi_{E} \sigma\right)$.

We may assume that $(0, \ldots, 0,1) \in E$. Let $\Lambda=\Lambda_{\varepsilon, \delta}$ denote the family of complex lines $L$ of the form

$$
L=\left\{\left(a_{1}, \ldots, a_{n-1}, \varepsilon\right) z+(0, \ldots, 0,1-\delta): z \in \mathbb{C}\right\},
$$

where $\sum\left|a_{i}\right|^{2}+\varepsilon^{2}=1$. There exist $\varepsilon_{0}, \delta>0$ such that for $0<\varepsilon<\varepsilon_{0}$, if $L \in \Lambda$, then the intersection $L \cap S \subset E$. Note that any two of these lines have only $(0,0, \ldots, 1-\delta)$ in common. This is the centre of all circles obtained by intersecting $S$ and $L$. Now take $\Lambda_{1}$ and $\Lambda_{2}$ to be disjoint subsets of $\Lambda$ such that $E_{i}=\bigcup_{L \in \Lambda_{i}} L \cap E$ is open for $i=1,2$ and $\operatorname{dist}\left(E_{1}, E_{2}\right)>0$. Take $g \in C(S)$ such that $g=1$ on $E_{1}$ and $g=0$ on $E_{2}$. Using the mean value property we obtain for pluriharmonic polynomials $P$

$$
\begin{aligned}
\int_{E}|g-P| d \sigma & \geq \int_{E_{1}} \ldots+\int_{E_{2}} \ldots \geq\left|\int_{E_{1}}(g-P) d \sigma\right|+\left|\int_{E_{2}}(g-P) d \sigma\right| \\
& =\left|\sigma\left(E_{1}\right)-c_{1} P(0, \ldots, 0,1-\delta)\right|+\left|c_{2} P(0, \ldots, 0,1-\delta)\right| .
\end{aligned}
$$

Here $c_{1}, c_{2}>0$ depend only on $E_{1}$ and $E_{2}$ respectively, and not on $P$. For the right hand side to be small, $P(0, \ldots, 0,1-\delta)$ will have to be close 
to $\sigma\left(L\left(E_{1}\right)\right) / c_{1}$ as well as to 0 , which is impossible.

Note that one could also give a proof like the one of Proposition 2.12, with $E_{i}$ for $A_{i}$. Also a proof can be given based on the fact that pluriharmonic polynomials are solutions of a system of tangential partial differential equations (cf. [R], Chap. 18). Then the same would be true for a limit in distribution sense on an open set, and a fortiori for a limit in $L^{1}(\sigma)$ sense, which would lead to a contradiction.

CONJECTURE. If $\mu$ is an extreme element in $M_{0} A(B)$, then $\mu \perp \sigma$.

Theorem 3.7 gives some support for this conjecture, while 3.3 shows that extreme measures can be concentrated on fairly large sets.

3.8. It is easy to see that a limit point of extreme points of a compact convex set need not be an extreme point. One can find an example in $\mathbb{R}^{3}$, namely, the convex hull of the union of a circle and an interval through a point of the circle, which is perpendicular to it. One might hope that the situation is better for $M_{0}$, but it is not, even if we consider convergence in the variation norm.

EXAMPLE. There exists a sequence $\left\{\mu_{j}^{C}\right\}$ in $M_{0} S$ which converges in variation norm to $\mu^{C} \in M_{0} S$, such that $\mu_{j}^{C}$ are all extreme but $\mu^{C}$ is not.

Again we work in $\mathbb{C}^{2}$, with coordinates $(z, w)$. Consider

$$
\begin{aligned}
\gamma_{j}= & \left\{z=e^{i \theta}:-1 / j<\theta<1 / j\right\} \\
& \cup\left\{z=e^{ \pm i / j}+t:-1 / 2 \leq t \leq 0,1 / 2 \leq|z| \leq 1\right\} \\
& \cup\left\{z=e^{i \theta} / 2: \alpha_{j} \leq|\theta| \leq \pi\right\},
\end{aligned}
$$

where $\alpha_{j} \approx 2 / j$ is such that $\gamma_{j}$ becomes a Jordan curve. Let $\mu_{j}$ be harmonic measure with respect to 0 on $\gamma_{j}$. One can verify immediately that $\mu_{j}$ tends to the representing measure $\mu$ for 0 on $\{|z|=1 / 2\}$ in variation norm. Then also $\mu_{j}^{C}$ tends in variation norm to $\mu^{C}$.

4. Push-forward measures. Another way to construct representing measures is by embedding analytic discs and pushing forward representing measures of subalgebras of the disc algebra. Consider a holomorphic map $\Phi=\left(\Phi_{1}, \ldots, \Phi_{n}\right): D \rightarrow B=B^{n}$, satisfying

(i) $\Phi(0)=0$,

(ii) $\Phi$ extends continuously to $\bar{D}$ and $\Phi(T) \subseteq S$.

For $\nu$ a measure on $T$ the push-forward $\Phi_{*}(\nu)$ of $\nu$ is defined by

$$
\int_{S} f(\zeta) d \Phi_{*}(\nu)=\int_{T}(f \circ \Phi)\left(e^{i \theta}\right) d \nu\left(e^{i \theta}\right) .
$$


It is obvious that $\Phi_{*}(d \theta / 2 \pi)$ is in $M_{0} S$. It is not true that $\Phi_{*}(d \theta / 2 \pi)$ is always extreme (cf. Example 4.3 below), but by a small variation of this construction one can construct extreme representing measures.

First assume, for simplicity, that $\left.\Phi\right|_{T}$ is injective. Let $A[\Phi]$ be the uniform subalgebra of $A(D)$ generated by the components $\Phi_{j}$ of $\Phi$. Then one easily checks that $\nu \in M_{0} A[\Phi]$ implies $\Phi_{*}(\nu) \in M_{0} S$.

If $\left.\Phi\right|_{T}$ is not injective, $A[\Phi]$ fails to separate points. In that case we regard $A[\Phi]$ as a uniform algebra on the compact Hausdorff space $T_{\Phi}:=T / \sim$, where $z \sim w$ iff $\Phi(z)=\Phi(w)$, and $T_{\Phi}$ is provided with the quotient topology. Now $\Phi$ induces a map $T_{\Phi} \rightarrow S$, and we can define $\Phi_{*}(\nu)$ as above, if $\nu$ is a measure on $T_{\Phi}$ (e.g., if $\nu \in M_{0} A[\Phi]$ ).

4.1. Proposition. Let $\nu \in M_{0} A[\Phi]$. Then $\Phi_{*}(\nu) \in M_{0} S$ is extreme if and only if $\nu$ is.

Proof. We restrict ourselves to the case where $\left.\Phi\right|_{T}$ is injective and leave the general case to the reader. First note that the map $\nu \rightarrow \Phi_{*}(\nu)$ is one-to-one on $M(T)$ : Suppose $\Phi_{*}(\nu)=0$. Let $g \in C(T)$ and $f \in C(S)$ be such that $f=g \circ \Phi^{-1}$ on $\Phi(T)$. Then $\langle g, \nu\rangle=\left\langle\Phi_{*}(\nu), f\right\rangle=0$, so that $\nu=0$. Hence, if $\nu \in M_{0} A[\Phi]$ is a nontrivial convex combination of $\nu_{1}$ and $\nu_{2}$, then $\Phi_{*}(\nu)$ is a nontrivial convex combination of $\Phi_{*}\left(\nu_{1}\right)$ and $\Phi_{*}\left(\nu_{2}\right)$.

Conversely, suppose that $\Phi_{*}(\nu)=\lambda \mu_{1}+(1-\lambda) \mu_{2}$, where $\mu_{1}, \mu_{2} \in M_{0} S$ and $0<\lambda<1$. For $g \in C(T)$, let $\widetilde{f}$ be any continuous extension to $S$ of the function $f=g \circ \Phi^{-1}$ on $\Phi(T)$. Define measures $\nu_{j} \in M(T)$ by

$$
\int_{T} g d \nu_{j}:=\int_{S} \tilde{f} d \mu_{j} .
$$

The right hand side is independent of the choice of the extension $\widetilde{f}$, since $\operatorname{Supp} \mu_{j} \subseteq \operatorname{Supp} \Phi_{*}(\nu) \subseteq \Phi(T)$. By definition, $\Phi_{*}\left(\nu_{j}\right)=\mu_{j}$. Also $\nu_{j} \in$ $M_{0} A[\Phi]$, because elements of $A[\Phi]$ can be uniformly approximated by functions $P \circ \Phi, P=P\left(z_{1}, \ldots, z_{n}\right)$ a holomorphic polynomial. Finally, $\nu=$ $\lambda \nu_{1}+(1-\lambda) \nu_{2}$, since $\Phi_{*}$ is injective.

4.2. $\mathrm{R}$ e $\mathrm{m}$ ark. The proof shows that the map $\Phi_{*}$ is a bijection of $M_{0} A[\Phi]$ onto the subset of $M_{0} S$ of representing measures whose support is contained in $\Phi(T)$.

4.3. ExAmple. We continue Example 2.13. Let $\Phi=\Phi(z)=(1 / \sqrt{2}) \times$ $\left(z^{2}, z^{3}\right): D \rightarrow B^{2}$. Then $A[\Phi]=\left\{f \in A(D): f^{\prime}(0)=0\right\}$. Each element of $M_{0} A[\Phi]$ is of the form $1+\operatorname{Re}\left(c e^{i \theta}\right) d \theta / 2 \pi$, with $c \in \mathbb{C}, 0 \leq|c| \leq 1$. In particular, $\Phi_{*}(d \theta / 2 \pi)$ is not extremal, since $d \theta / 2 \pi$ is not extremal in $A[\Phi]$. However, $\Phi_{*}(d \theta / 2 \pi)$ has minimal support.

One could hope that convex combinations of push-forward measures $\Phi_{*}(\nu)$ are weak*-dense in $M_{0} S$, but this is probably false. However, one 
has the following weaker result, which identifies the closed linear subspace Span $M_{0} S \subseteq M(S)$ spanned by $M_{0} S$.

4.4. Theorem. For $k, l \in \mathbb{N}$, let $\Phi_{k, l}: D \rightarrow B$ be given by $\Phi_{k, l}(z)=$ $(1 / \sqrt{2})\left(z^{k}, z^{l}, 0, \ldots, 0\right)$, and let $\mathcal{U}(N)$ be the unitary group. Then

$$
\operatorname{Span}\left\{\left(U \circ \Phi_{k, l}\right)_{*}(d \theta / 2 \pi): k, l \in \mathbb{N} \backslash\{0\},(k, l)=1, U \in \mathcal{U}(n)\right\}
$$

is weak ${ }^{*}$-dense in $\operatorname{Span} M_{0} S$ (here, $(k, l)=$ g.c.d. $\left.(k, l)\right)$.

Proof. Note that $\Phi_{k, l}(0)=0$ iff neither $k$ nor $l$ are 0 , so then $\Phi_{k, l}$ satisfies (4.1). The dual of $M(S)$ (with the weak ${ }^{*}$-topology) can be identified with $C(S)$, if we identify $g \in C(S)$ with the linear functional $\mu \rightarrow\langle g, \mu\rangle$. Let $g \in C(S)$ be such that

$$
\begin{aligned}
& \int_{S} g\left(U \circ \Phi_{k, l}\right)_{*}(d \theta / 2 \pi)=0, \\
& \forall k, l \in \mathbb{N} \backslash\{0\},(k, l)=0 \text { and } \forall U \in \mathcal{U}(n) .
\end{aligned}
$$

We claim that (4.3) implies that $g \in \mathrm{PH}(S)$, that is, by definition, the harmonic extension of $g$ is pluriharmonic. This will imply that for all $\mu \in$ $M_{0} S, \int g d \mu=g(0)=0$, by (4.3), and the theorem follows by Hahn-Banach.

Let $\Pi_{r, s}$ denote the orthogonal projection onto the space $H(r, s)$ of spherical harmonics of bi-degree $(r, s)$ (cf. [R], Chap. 12). Thus, to prove the claim, it suffices to show that $\Pi_{r, s} g=0$ if $r s \neq 0$. Let $Y$ be the set of all $g \in C(S)$ satisfying (4.3). Note that $Y$ is a closed, $\mathcal{U}(n)$-invariant subspace of $C(S)$. In this situation Theorem 12.3.6 in [R] states that $\Pi_{r, s} Y \neq\{0\}$ implies $H(r, s) \subseteq Y$. However, $H(r, s) \nsubseteq \subseteq Y$ if $r s \neq 0$, since $z_{1}^{r} \bar{z}_{2}^{s} \in H(r, s)$ and if we take $k=s /(r, s), l=r /(r, s)$, we find

$$
\int_{S} z_{1}^{r} \bar{z}_{2}^{s}\left(\Phi_{k, l}\right)_{*}\left(\frac{d \theta}{2 \pi}\right)=\int_{T} \zeta^{r k} \bar{\zeta}^{s l} \frac{d \theta}{2 \pi}=1 .
$$

One of the motivations for studying $M_{0} S$ is the Cole-Range theorem, which implies that each element in $A(S)^{\perp}$ is absolutely continuous with respect to some measure in $M_{0} S$. We give a description of $A(S)^{\perp}$ in the spirit of Theorem 4.4 .

4.5. TheOREM. Let $\mathcal{P}_{0}$ denote the space of holomorphic polynomials on $\mathbb{C}$ which vanish at the origin. Then

$$
\begin{aligned}
& \operatorname{Span}\left\{\left(\Phi_{k, l} \circ U\right)_{*}\left(P\left(e^{i \theta}\right) d \theta\right): k, l \in \mathbb{N} \backslash\{0\}, U \in \mathcal{U}(n)\right\} \\
& \text { is weak*-dense in } A(S)^{\perp} \text {. }
\end{aligned}
$$

Pro of. Note that if $\Phi: D \rightarrow B$ is holomorphic and extends continuously to $\bar{D}$, then $\nu \in A(T)^{\perp}$ implies $\Phi_{*}(\nu) \in A(S)^{\perp}$. Let $Y$ be the subspace of all $g \in C(S)$ which are annihilated by the measures in (4.4). It suffices to prove that $Y \subseteq A(S)$. As before, $Y$ is $\mathcal{U}(n)$-invariant. Suppose $\Pi_{r, s} \neq 0$ for 
some $(r, s)$ with $s \neq 0$. Then $H(r, s) \subseteq Y$ and in particular, for all $m \geq 1$, and all $k, l \in \mathbb{N}$,

$$
0=\int_{S} z_{1}^{r} \bar{z}_{2}^{s}\left(\Phi_{k, l}\right)_{*}\left(e^{i m \theta} \frac{d \theta}{2 \pi}\right)=\int_{0}^{2 \pi} e^{i(k r+m-l s) \theta} \frac{d \theta}{2 \pi} .
$$

The latter expression equals 1 for a suitable choice of $m, k, l$, which is a contradiction.

Remark. The same proof shows that $\operatorname{Span}\left\{\left(\Phi_{0,1} \circ U\right)_{*}\left(P\left(e^{i \theta}\right) d \theta\right)\right\}$ is also weak* ${ }^{*}$-dense in $A(S)^{\perp}$, but note that $\Phi_{0,1}(D)$ does not meet the origin.

\section{References}

[A] E. M. Alfsen, Compact Convex Sets and Boundary Integrals, Ergeb. Math. Grenzgeb. 57, Springer, Berlin 1971.

[BSZ] L. Brown, A. Shields and K. Zeller, On absolutely convergent exponential sums, Trans. Amer. Math. Soc. 96 (1960), 162-183.

[D] R. G. Douglas, On extremal measures and subspace density, Michigan Math. J. 11 (1964), 243-246.

[G] T. Gamelin, Uniform Algebras, Prentice-Hall, Englewood Cliffs, N.J., 1969.

[Gar] J. B. Garnett, Bounded Analytic Functions, Academic Press, New York 1981.

[HR] K. Hoffman and H. Rossi, Extensions of positive weak ${ }^{*}$-continuous functionals, Duke Math. J. 37 (1967), 453-466.

[JW] P. Jones and T. Wolff, Hausdorff dimension of harmonic measures in the plane, Acta Math. 161 (1989), 131-144.

[K] P. Koosis, Introduction to $H_{p}$ Spaces, London Math. Soc. Lecture Note Ser. 40, Cambridge Univ. Press, 1980.

[Ø] B. Øksendal, Null sets for measures orthogonal to $R(X)$, Amer. J. Math. 94 (1972), 331-342.

[P] R. R. Phelps, Lectures an Choquet's Theorem, van Nostrand Math. Stud. 7, van Nostrand, Princeton 1966.

[R] W. Rudin, Function Theory in the Unit Ball of $\mathbb{C}^{n}$, Grundlehren Math. Wiss. 241, Springer, Berlin 1980.

[Ry] J. Ryff, The support of representing measures for the disc algebra, in: Function Algebras, F. Birtel (ed.), Scott, Foresman and Company, Glenview 1966.

DIVISION DE MATEMÁTICAS

FACULTAD DE CIENCIAS

UNIVERSIDAD AUTÓNOMA DE MADRID

28049 MADRID, SPAIN
FACULTEIT WISKUNDE EN INFORMATICA UNIVERSITEIT VAN AMSTERDAM PLANTAGE MUIDERGRACHT 24 1018 TV AMSTERDAM, THE NETHERLANDS 